\title{
Shear bond strengths of three glass ionomer cements to enamel and dentine
}

\author{
Thiago-Saads Carvalho ${ }^{1}$, Willem-Evert van Amerongen ${ }^{2}$, Anton de Gee ${ }^{2}$, Marcelo Bönecker ${ }^{3}$, Fábio-Correia \\ Sampaio $^{4}$
}

\author{
${ }^{1}$ Departamento de Odontologia Clínica e Social, Universidade Federal da Paraíba, João Pessoa PB; Departamento de Odontope- \\ diatria, Faculdade de Odontologia da USP. Brazil \\ ${ }^{2}$ Academic Centre for Dentistry Amsterdam - ACTA, Amsterdam, the Netherlands \\ ${ }^{3}$ Departamento de Odontopediatria, Faculdade de Odontologia da USP. Brazil \\ ${ }^{4}$ Departamento de Odontologia Clínica e Social, Universidade Federal da Paraíba, João Pessoa PB, Brazil
}

Correspondence:

Departamento de Odontopediatria

Faculdade de Odontologia da Universidade de São Paulo (FOUSP)

Av. Prof. Lineu Prestes, 2227. Cidade Universitária

05508-000 São Paulo-SP Brazil

thiagosaads@hotmail.com

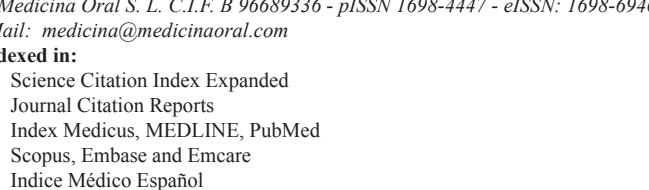

\begin{abstract}
Objectives: The shear bond strength of three glass ionomer cements (GIC) to enamel and dentine was evaluated. Study Design: Sound permanent human molars $(n=12)$ were grinded perpendicular to their axial axes, exposing smooth, flat enamel and dentine surfaces. The teeth were embedded in resin and conditioned with polyacrylic acid (25\%; 10s). Twenty four specimens of each GIC: Fuji IX (FJ - GC), Ketac Molar Easymix (KM - 3M ESPE) and Maxxion (MX - FGM) were prepared according to the Atraumatic Restorative Treatment (ART) (12 enamel and 12 dentine), in a bonding area of $4.91 \mathrm{~mm} 2$ and immersed in water $\left(37^{\circ} \mathrm{C}, 24 \mathrm{~h}\right)$. The shear bond strength was tested in a universal testing machine. Non-parametric statistical tests (Friedman and post-hoc Wilcoxon Signed Ranks) were carried out $(\mathrm{p}=0.05)$.

Results: The mean $( \pm \mathrm{sd})$ of shear bond strength $(\mathrm{MPa})$, on enamel and dentine, were: KM $(6.4 \pm 1.4$ and $7.6 \pm 1.5)$, FJ (5.9 \pm 1.5 and $6.0 \pm 1.9)$ and MX (4.2 \pm 1.5 and $4.9 \pm 1.5)$, respectively. There was a statistically significant difference between the GICs in both groups: enamel $(p=0.004)$ and dentine $(p=0.002)$. The lowest shear bond value for enamel was with MX and the highest for dentine was KM ( $\mathrm{p}<0.05)$.

Conclusion: It is concluded that KM has the best adhesion to both enamel and dentine, followed by FJ and MX.
\end{abstract}

Key words: Shear strength, glass ionomer cements, enamel, dentine. 


\section{Introduction}

Glass ionomer cements (GIC) were introduced in the 1960s, and since then their clinical applications have been improved. This was mainly due to their chemical bonding to tooth hard tissues, biocompatibility, fluoride release, as well as their ability to influence the remineralization of carious lesions $(1,2)$.

Improved GIC, with higher powder: liquid ratios, may have enhanced mechanical properties. Such glass ionomers are indicated for atraumatic restorative treatments (ART), which is patient friendly, and causes less discomfort for the patients (3). GICs have enhanced properties of the highly viscous glass ionomer cements are based on improved chemistry (4). However, some GIC restorations may still be susceptible to wear and fracture.

New improvements to GICs are frequently put on the market, amplifying the spectrum of materials used for restoration. These relatively new materials, such as Maxxion (FGM), still need to undergo additional tests, such as adhesion to the tooth structure, to further explore their clinical applications. This is of particular importance, especially for developing countries, to amplify the use of such materials and, therefore, broaden the applications of the atraumatic restorative treatment. Therefore, the aim of this study was to compare the shear bond strength to enamel and dentin, of three highly viscous glass ionomer cements: Fuji IX (GC, Europe), Ketac Molar Easymix (3M-ESPE, Germany) and Maxxion (FGM, Brazil).

\section{Material and Methods \\ Sample preparation}

Enamel and dentine samples were prepared. Freshly extracted non-carious unrestored permanent human molars $(n=12)$ were selected for the shear bond tests. The roots were removed perpendicular to the long axis of the tooth, using a low-speed diamond-disk with water coolant. The teeth occlusal surfaces were grinded flat using 320, 600 and 1200-grit silicon carbide abrasive paper, under constant running water, on a polishing machine (Buehler Ecomet V, Buehler Ltd, Lake Bluff, IL, USA), in order to obtain flat smooth enamel surfaces. The dentine surfaces were also grinded, as described, to obtain $\pm 5 \mathrm{~mm}$ tooth disks, containing enamel on one side, and dentine on the other. The tooth samples were then mounted in poly(methyl methacrylate) resin.

Preparation of Glass Ionomer Cements

Standard polyethylene disks with a $4.91 \mathrm{~mm}^{2}$ orifice were clamped onto the tooth samples and were used as molds. The GIC "restorations" were prepared while the teeth were kept in a warm and humid environment, generated by a water bath at $37^{\circ} \mathrm{C}$. The tooth samples were placed on a support such that the upper part of the samples was above the waterline, exposing a dry surface to carry out the restorations. The lower part of the samples was still in contact with the warm water, which maintained the temperature of the sample.

The surfaces were conditioned with the polyacrylic acid Ketac Conditioner (3M ESPE) for 10 seconds. It was then washed with three cotton pellets dipped in water, at room temperature, then dried with three clean pellets, according to ART procedure (5).

The GICs tested were Fuji IX (FJ, GC corporation, Japan), Ketac Molar Easymix (KM, 3M ESPE, Germany) and Maxxion R (MX, FGM, Brazil). All GIC restorations were prepared according to the manufacturer's instructions. The hand mixing time was standardized at 30 seconds for all GICs. In case of overfilling, the excess was removed immediately using a carver. A humid paper towel was placed over the samples after $1 \frac{1}{2}$ minutes to keep the teeth moist.

A time of $3 \frac{1}{2}$ minutes was allowed for the GIC initial setting, and then the samples were placed completely under water. The clamps and polyethylene disks were removed after 20 minutes. The samples were kept in the water bath at $37^{\circ} \mathrm{C}$ for 24 hours.

The tooth samples were reused after the shear bond test by removing the glass ionomer remnants by wet grinding.

\section{Shear bond test}

The samples were placed vertically on a support to shear off the specimen. Each GIC specimen was loaded in a universal testing machine (ACTA Intense, the Netherlands) with a cross-head speed of $0.5 \mathrm{~mm} / \mathrm{min}$. The force, in Newton $(\mathrm{N})$, required to displace the GIC restoration was recorded and then the bond strength (Megapascal [MPa]) was calculated by dividing the shear force $(\mathrm{N})$ by the area of adhesion $\left(4.91 \mathrm{~mm}^{2}\right)$.

After the shear test, all tooth samples were examined under a light microscope for remnant parts of glass ionomer.

A total of 24 specimens of each GIC were tested, 12 on enamel and 12 on dentine.

Statistical analysis

The values for the bond strengths were only accepted in a range of $[(\mathrm{x}-2 \delta) \leq \mathrm{n} \leq(\mathrm{x}+2 \delta)]$ to maintain the 0.05 significance. A normal distribution test for the values was carried out. Friedman's test was used to find possible significant differences between the GIC in the enamel and dentine groups. A post-hoc Wilcoxon Signed Ranks test was later performed to find possible differences between the materials in the same group, and within each glass ionomer in both enamel and dentine.

\section{Results}

When observed under the light microscope, almost all fractures were largely cohesive in the glass ionomer, in both groups, enamel and dentine (Table 1).

The GIC that had the highest mean bond strength to 
Table 1. Mean shear bond strengths (MPa) and standard deviation (SD) to enamel and dentine for different glass ionomer cements.

\begin{tabular}{|c|c|c|c|c|}
\hline \multirow{2}{*}{ Tooth Surface } & $\begin{array}{c}\text { Glass } \\
\text { Ionomer }\end{array}$ & Fuji IX & $\begin{array}{c}\text { Ketac Molar } \\
\text { Easymix }\end{array}$ & Maxxion \\
\hline \multirow{2}{*}{ Enamel } & Mean & $5.9^{\mathrm{a}}$ & $6.4^{\mathrm{a}}$ & $4.2^{\mathrm{b}}$ \\
\cline { 2 - 5 } & $(\mathrm{SD})$ & $(1.5)$ & $(1.4)$ & $(1.5)$ \\
\hline \multirow{2}{*}{ Dentine } & Mean & $6.0^{\mathrm{a}}$ & $7.6^{\mathrm{b}}$ & $4.9^{\mathrm{a}}$ \\
\cline { 2 - 5 } & (SD) & $(1.9)$ & $(1.5)$ & $(1.5)$ \\
\hline
\end{tabular}

${ }^{a}$ Different small letters denote statistically significant difference $(\mathrm{p}<0.05)$ between glass ionomers in the same group (rows).

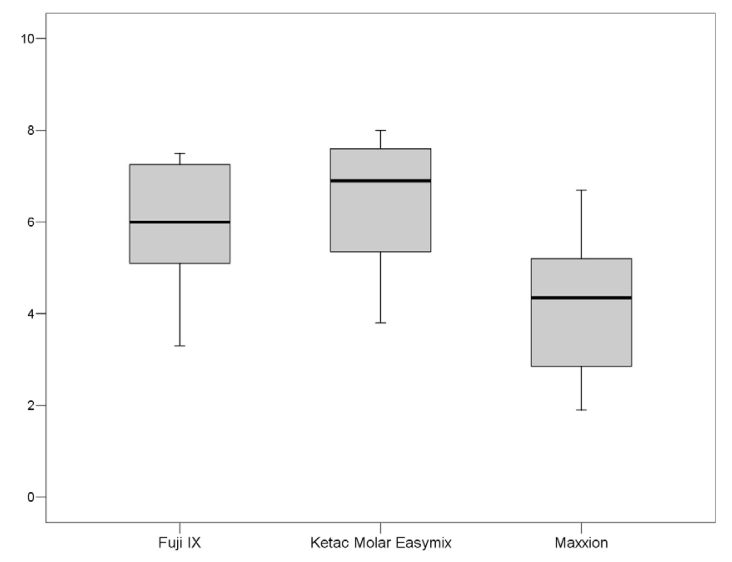

Fig. 1. Shear bond strength distribution (median and range) of the glass ionomer cements to enamel.

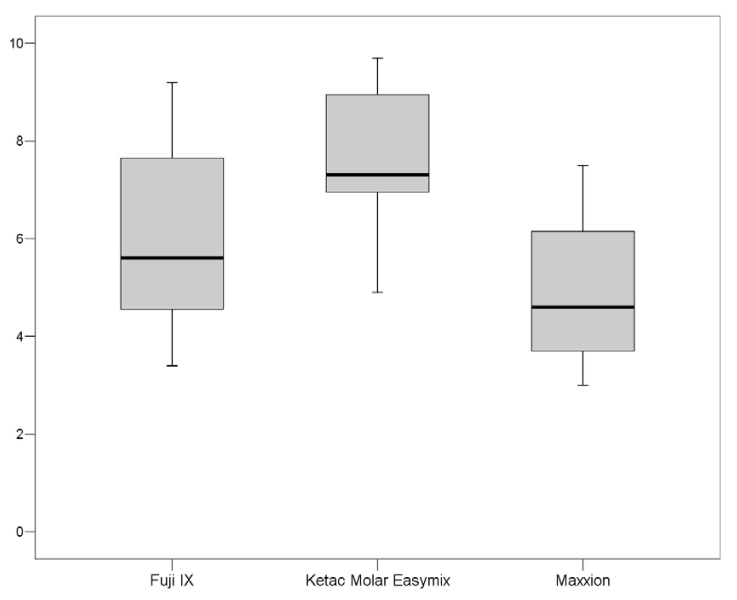

Fig. 2. Shear bond strength distribution (median and range) of the glass ionomer cements to dentine. both enamel and dentine was Ketac Molar Easymix, followed by Fuji IX and Maxxion. All GICs had higher bond strength values in dentine, however, this was only significant for KM.

The data did not present a normal distribution (Fig. 1, 2), therefore, Friedman's test, and a post-hoc Wilcoxon Signed Ranks test, were carried out. Statistically significant differences were found between the GICs' bond strengths to enamel $(p=0.005)$ and dentine $(p=0.011)$ as demonstrated in table 1 .

\section{Discussion}

The glass ionomers mainly used for ART restorations have high powder: liquid ratios and, therefore, higher compressive strengths $(6,7)$. This study followed other results presented in the literature, which established that the GICs have higher bond strength to enamel, than to dentine $(8,9)$. Extensive tests have been carried out using Fuji IX, but Ketac Molar Easymix and Maxxion are relatively new, and although some tests have been carried out (10), further mechanical tests are still required for their use as ART restoration material.

In order to test these glass ionomer cements for future field trials, the methods were carried out using the ART protocol, thus justifying the use of cotton pellets for washing and drying the restoration areas (5). Although the protocol also suggests placing a protective layer of petrolatum over the GIC restorations, to test the different cements on enamel and dentine using the same tooth specimen, the samples used in this study were not covered with petroleum jelly. Such a layer could contaminate the tooth samples with traces of organic compounds, reducing the chemical adherence of the different cements to the tooth structures.

Similarly, the GIC samples were kept in a $37^{\circ} \mathrm{C}$ water bath, and despite the fact that samples stored in oil have greater mechanical strength than those stored in water (10), lubricant storage or coating was not possible due to the re-use of the tooth samples. It has been observed that storage in water causes softening of the cement's outer layer $(11,12)$, but hardness values increase with storage time due to migration of ions from the core to the surface, suggesting that samples more than $1 \mathrm{~mm}$ in depth are not vulnerable to storage fluids (11). Therefore, it could be suggested that the bonding interface between the GIC and the enamel, or dentine, would not be greatly influenced by the surrounding water. On the other hand, if the results in this study were to have been influenced by the storage in water, we may suppose that bonding values would be higher in ART restorations in field applications, where a lubricant coating is recommended. Also, although petroleum jelly coating reduces the water uptake by the GICs by fifty percent, it has been suggested that nail varnish is a better protective agent (13). 
Studies testing shear bond strengths of GICs to dentine have found values ranging from 1.32-4.10 $(14,15)$. In our study, these values were higher, in the range of 4.9-7.6 $\mathrm{MPa}$, which may represent some improvement on the highly viscous GICs developed for ART. Higher bond strengths (12-15 $\mathrm{MPa}$ ) using microtensile bond testing were reported. However, these high values reflect the use of samples with a smaller interface area between the GIC and dentine (16).

In order to standardize the re-use of the teeth samples, grinding was repeated after each experiment to remove the layer of the GIC previously tested. Thus a clean smooth surface for new tests was obtained. As a result of sample grinding between tests, the dentine surface used for subsequent adhesion would be a few micrometers closer to the enamel-dentine junction. However the grinding would not affect the results and some points support this argument: First, all GIC adhesion tests to dentine were carried out in the outer half of the dentine whereas adhesion tests to enamel were carried out in the inner half of the enamel. Second, the calcium and mineral content in the middle area of the dentine shows only small variations. The calcium/mineral content decreases in dentine only in the region within $400 \mu \mathrm{m}$ from the dentine-enamel junction (DEJ) (17). As the GICs form a chemomechanical bond with the calcium and mineral content present in the tooth structure, and this content varies in dentine only when very close to the DEJ, it may be suggested that the adhesion values found in the present study did not vary so much due to the mineral/ calcium content of the grinded dentine. Thus, such small differences in depth of the dentine could have caused only small variations in the adhesion values.

In in vivo restorations, the GICs are mainly used to restore carious dentine lesions and to seal fissures (5). Hence, the restorations performed in ART field trials remain in place because of adhesion to dentine, as well as to enamel. Also, the GIC restoration bonds not only to dentine on the cavity floor, but also on the walls of the cavity. This possibly covers a complete range of prism structure. Moreover, if the bond strength is greater when the tubules are parallel to the bonded interface than when the tubules are cut perpendicularly (18), it may be suggested that, in clinical situations, the bond strength is much greater on the cavity walls than on the cavity floor.

It is important to state that the samples were kept in water throughout the experiment. It has been observed that in vivo dentine has tubules mainly filled with dentinal fluid and odontoblastic processes, so the use of water as a means of storage may have altered the permeability of the dentine, thus causing potential differences between in vitro studies and in vivo results (19). Such a lack of vitality of dentine in in vitro studies may limit the possibility of extrapolating these findings to in vivo situations.
In accordance to previous studies $(1,8)$, most fractures analyzed under the light microscope after the shear tests were largely cohesive rather than adhesive. This may be due to the low resistance to early wear, and the formation of glass ionomer matrix (20). Therefore, part of the glass ionomer remained adhered to the tooth structures, while part was broken at the interface GIC-tooth. This suggests that the bond strengths between the GICs and the tooth could be greater than the present results, also due to the chemical bonding (9). In spite of suggestions by Mitra (1), as well as Kleverlaan et al. (10), that the use of light cured glass ionomers or the use of heat supply from light curing devices increases the overall cohesive strength of the materials, ART is intended to enable dentists to carry out restorations in areas distant from electrical sources. Therefore, the use of light cured glass ionomer cements or heat supplying devices to increase success rates of the restorations may not be globally viable.

Inverse correlation between mechanical strength and fluoride release has been previously described in the literature, suggesting that materials exhibiting high fluoride release values have poorer mechanical properties (7). Therefore, the glass ionomer Maxxion (FGM) could have a greater fluoride release than the other glass ionomer cements, which could explain the lower values observed in this study. On the other hand, GICs have shown to remineralize - or even hypermineralize dentine lesions, possibly due to fluoride that is released from the cement restorations and retained on the cariogenic biofilm (2).

Although Maxxion had lower shear bond values than Fuji IX and Ketac Molar Easymix on both enamel and dentine, in in vivo ART restorations, a better performance could be expected if satisfactory surface protection is carried out, thus lessening water absorbance. Nevertheless, more tests using this cement are required, and more research on this topic ought to be carried out.

\section{References}

References with links to Crossref - DOI

1. Mitra SB. Adhesion to dentin and physical properties of a lightcured glass-ionomer liner/base. J Dent Res. 1991;70:72-4.

2. Ten Cate JM, Van Duinen RN. Hypermineralization of dentinal lesions adjacent to glass-ionomer cement restorations. J Dent Res. 1995;74:1266-71.

3. Carvalho TS, Ribeiro TR, Bönecker M, Pinheiro EC, Colares V. The atraumatic restorative treatment approach: an "atraumatic" alternative. Med Oral Patol Oral Cir Bucal. 2009;14:e668-73.

4. Yap AU, Pek YS, Cheang P. Physico-mechanical properties of a fast-set highly viscous GIC restorative. J Oral Rehabil. 2003;30:1-8. 5. Frencken JE, Pilot T, Songpaisan Y, Phantumvanit P. Atraumatic restorative treatment (ART): rationale, technique, and development. J Public Health Dent. 1996;56:135-40.

6. Rutar J, McAllan L, Tyas MJ. Three-year clinical performance of glass ionomer cement in primary molars. Int J Paediatr Dent. 2002;12:146-7.

7. Xu X, Burgess JO. Compressive strength, fluoride release and recharge of fluoride-releasing materials. Biomaterials. 2003;24:245161 . 
8. Powis DR, Follerås T, Merson SA, Wilson AD. Improved adhesion of a glass ionomer cement to dentin and enamel. J Dent Res. 1982;61:1416-22.

9. Lin A, McIntyre NS, Davidson RD. Studies on the adhesion of glass-ionomer cements to dentin. J Dent Res. 1992;71:1836-41.

10. Kleverlaan CJ, Van Duinen RN, Feilzer AJ. Mechanical properties of glass ionomer cements affected by curing methods. Dent Mater. 2004;20:45-50.

11. Dupuis V, Moya F, Payan J, Bartala M. Depth microhardness of glass ionomer cements. Biomaterials. 1996;17:71-4.

12. De Moor RJ, Verbeeck RM. Changes in surface hardness of conventional restorative glass ionomer cements. Biomaterials. 1998:19:2269-75.

13. Serra MC, Navarro MF, Freitas SF, Carvalho RM, Cury JA, Retief DH. Glass ionomer cement surface protection. Am J Dent. 1994;7:203-6.

14. Hewlett ER, Caputo AA, Wrobel DC. Glass ionomer bond strength and treatment of dentin with polyacrylic acid. J Prosthet Dent. 1991;66:767-72.

15. Burke FM, Lynch E. Glass polyalkenoate bond strength to dentine after chemomechanical caries removal. J Dent. 1994;22:283-91. 16. Yip HK, Tay FR, Ngo HC, Smales RJ, Pashley DH. Bonding of contemporary glass ionomer cements to dentin. Dent Mater. 2001;17:456-70

17. Tesch W, Eidelman N, Roschger P, Goldenberg F, Klaushofer K, Fratzl P. Graded microstructure and mechanical properties of human crown dentin. Calcif Tissue Int. 2001;69:147-57.

18. Ogata M, Okuda M, Nakajima M, Pereira PN, Sano H, Tagami J. Influence of the direction of tubules on bond strength to dentin. Oper Dent. 2001;26:27-35.

19. Ozok AR, Wu MK, Ten Cate JM, Wesselink PR. Effect of dentinal fluid composition on dentin demineralization in vitro. J Dent Res. 2004;83:849-53.

20. De Gee AJ, Van Duinen RN, Werner A, Davidson CL. Early and long-term wear of conventional and resin-modified glass ionomers. J Dent Res. 1996;75:1613-9.

\section{Acknowledgements}

Our appreciation is going out to Mr. A. Werner who was of great help in performing the tests in the lab and the manufacturers of Fuji IX (GC), Ketac Molar Easy-Mix (3M-ESPE) and Maxxion (FGM) who supplied the materials for this study. Research with financial support from CAPES. 